\title{
Magnetotransport properties of oxidized iron thin films
}

\author{
Jolanta Stankiewicz, ${ }^{1, *}$ Félix Jiménez-Villacorta, ${ }^{2}$ and Carlos Prieto ${ }^{2}$ \\ ${ }^{1}$ Instituto de Ciencia de Materiales de Aragón, Consejo Superior de Investigaciones Científicas and Universidad de Zaragoza, \\ 50009-Zaragoza, Spain \\ ${ }^{2}$ Instituto de Ciencia de Materiales de Madrid, Consejo Superior de Investigaciones Científicas, Cantoblanco, 28049-Madrid, Spain
}

(Received 22 June 2005; revised manuscript received 13 December 2005; published 27 January 2006)

\begin{abstract}
We report results of electrical resistivity, Hall effect, and anisotropic magnetoresistance (AMR) measurements performed on thin films $(\sim 80 \mathrm{~nm})$ of naturally oxidized iron in the 5 to $300 \mathrm{~K}$ temperature range, and in magnetic fields up to $5 \mathrm{~T}$. The films were grown at low temperatures. We find that the resistivity, coercive field, as well as the magnetoresistance of the films are affected by growth conditions, particularly by the substrate temperature which controls the size and shape of crystalline grains. We used AMR as a probe for magnetization reversal studies in our films. We find that the coercive fields vary as $\sqrt{T}$ as in systems of weakly interacting ensembles of magnetic nanoparticles even though dipole-dipole interactions may well play a significant role.
\end{abstract}

DOI: $10.1103 /$ PhysRevB.73.014429

PACS number(s): 75.70.Ak, 73.50.Jt, 73.61.At

\section{INTRODUCTION}

Nanoscopic magnetic particles are attractive for a variety of applications which span from magnetic recording to medical diagnostic and drug delivery. Their physical properties are not the less fascinating because of finite size effects. Novel phenomena, different from the bulk behavior, are observed as the size of particles shrinks to nanometer range. Many of these phenomena vary with shape and microstructure. ${ }^{1}$ Magnetic properties are the ones most extensively investigated in different systems of fine particles while electrical transport has been mainly studied in multilayered or granular materials. In spite of many published results, the relation between morphology, magnetic state and current conduction in magnetic nanosystems has not yet been clearly established. Here, we report electrical transport properties of a relatively simple nanosystem which is made up of iron thin films grown at low temperatures. We find that the properties of such system vary with growth temperature in a reproducible and systematic way. As expected, they are directly related to the magnetic state of the films. This allows us to use anisotropic magnetoresistance for probing magnetization dynamics in our system. It is our aim to elucidate the role played by various contributions to the electrical and magnetic properties of the system we have studied.

The reciprocal effects between charge carriers and the magnetic state of the system in which conduction takes place arise from spin-orbit coupling. It gives rise to anisotropic magnetoresistance (AMR) and the anomalous Hall effect (AHE). Mixing of spin-up and spin-down $d$ states by spinorbit interaction leads to magnetization-direction dependent $s-d$ scattering. ${ }^{2-4}$ Consequently, one expects the resistivity to become anisotropic in the magnetic field. ${ }^{3}$ The resistivity varies with angle $\theta$, between the current direction and the magnetization, as $\rho(\theta)=\rho_{\perp}+\left(\rho_{\|}-\rho_{\perp}\right) \cos ^{2}(\theta)$. Here $\rho_{\perp}$ and $\rho_{\|}$ are the resistivity for fields that are applied perpendicular and parallel to the current $\mathbf{I}$, respectively. Sweeping the external magnetic field through the range $-H_{\text {sat }} \leqslant H \leqslant H_{\text {sat }}\left(H_{\text {sat }}\right.$ is the magnetization saturation field) gives rise to a maximum or minimum in the resistivity, depending on the angle $\theta$. The observed maxima or minima correspond quite well to the coercive field $H_{c}$. AMR values of a few percent are obtained in thin magnetic films. Therefore, the AMR measurements provide relatively easy and fast method of obtaining hysteresis loops.

On the other hand, the anomalous Hall effect arises from asymmetric scattering of the current carriers which are subject to spin-orbit interactions. In itinerant magnetism models, skew scattering of charge carriers leads to the relation $\rho_{H} \propto \rho\langle M\rangle$, where $\rho_{H}$ is the Hall resistivity, $\rho$ is the total resistivity, and $M$ is the system magnetization..$^{5}$ In addition to skew scattering, a nonclassical effect of a lateral displacement (side jump) of electrons at every scattering event, gives a quadratic variation of $\rho_{H}$ with $\rho .{ }^{6}$ The latter mechanism can be important in the AHE of concentrated alloys. There is also an intrinsic contribution to the AHE, originally derived by Karplus and Luttinger, who have shown how spin-orbit interaction, in addition to the effect of interband coupling, gives rise to an anomalous velocity and transverse current in ferromagnetic materials with no need of scattering. ${ }^{7}$ This contribution gives $\rho_{H} \propto \rho^{2}$. The importance of the various mechanisms responsible for the AHE in magnetic systems has not yet been clearly established. Phenomenologically, the Hall resistivity is given by $\rho_{H}=R_{o} \mathbf{B}+R_{S} 4 \pi \mathbf{M}$, where $R_{o}$ is the ordinary Hall coefficient, $R_{s}$ is the AHE coefficient, $\mathbf{B}$ is the applied magnetic induction, and $\mathbf{M}$ is the spontaneous magnetization. In ferromagnetic samples, $R_{s}$ is much larger than $R_{o}$, and $\rho_{H} \propto R_{S} M$. Therefore, AHE effect measurements, give information about the magnetization behavior and important scattering mechanisms in magnetic materials. We use both AMR and AHE effects to study oxidized Fe films.

\section{EXPERIMENT}

Thin films of ultrafine particles can be prepared by various methods, such as thermal evaporation, electron beam deposition, or ion beam sputtering. Cooling of the substrate during the deposition process improves the versatility of 
these methods significantly. In particular, the size and shape of crystalline grains in thin films can be modified and controlled by varying the growth temperature. Here, we explore the electrical transport properties of thin films prepared by sputtering of $\mathrm{Fe}$ on naturally oxidized $\mathrm{Si}(100)$ substrates whose deposition temperature $T_{s}$ has been varied between 170 and $300 \mathrm{~K}$. The films, typically $80-\mathrm{nm}$ thick, were slightly oxidized by exposing them to air at room temperature for $30 \mathrm{~min}$ (due to experimental restrictions), and subsequently they were capped with a $\mathrm{SiO}_{2}$ layer in order to prevent further oxidation. X-ray diffraction and absorption experiments show that crystalline grain size in the films decreases as the substrate temperature decreases. The films exhibit a (110)-bcc texture. Samples prepared at room temperature have elongated grains coming from columnar growth while those prepared at $\$ 200 \mathrm{~K}$ have more spherical grains. The in-plane size of crystalline grains in our films is approximately $30 \AA$, independent of growth temperature. The outof-plane (perpendicular to the film) crystalline-grain size decreases from about 90 to $\sim 60 \AA$ as $T_{s}$ varies from 300 to $170 \mathrm{~K}^{8}$ Therefore, growth at lower temperatures gives rise to less elongated and smaller crystal grains which are, therefore, more easily oxidized.

We have measured the electrical resistivity, magnetoresistance and Hall effect of the films in a 4 to $300 \mathrm{~K}$ temperature range and in external magnetic fields up to $5 \mathrm{~T}$. The measurements were performed on approximately $5 \mathrm{~mm}^{2}$ square shaped samples in a in-plane (applied magnetic field in the plane of the sample) and out-of-plane (applied magnetic field perpendicular to the sample) geometry. We used the van der Pauw method for the resistivity measurements. Indium contacts were placed at the corners of the samples. The magnetoresistance measurements were carried out with a lowfrequency in-plane current of $\approx 50 \mu \mathrm{A}$. Magnetization measurements were performed with a commercial superconducting quantum interference device (SQUID) magnetometer. From here on, we assume that effects related to the surface of the films are negligible. Surface scattering can drastically affect transport properties of thin films. However, in homogeneous films, $80 \mathrm{~nm}$ thick, these effects are expected to be unimportant. ${ }^{4,9}$

\section{RESULTS AND DISCUSSION}

\section{A. Resistivity and Hall effect}

How the resistivity of the oxidized Fe films varies with temperature is shown in Fig. 1. The resistivity is metallic in all the samples we studied. However, a weak upturn in the resistivity is observed below $T \leq 30 \mathrm{~K}$ in films prepared at $T_{s}=200$ and $170 \mathrm{~K}$, which might arise from intergrain tunneling. On the other hand, $\rho$, as well as the residual resistivity of the films, decreases strongly as $T_{s}$ increases (see the inset of Fig. 1). The microstructural studies (x-ray diffraction and $\mathrm{x}$-ray absorption spectroscopy) we have performed on the same films show that crystalline-grain size increases as $T_{s}$ increases. ${ }^{8,10}$ In addition, Rutherford backscattering spectroscopy measurements show more effective oxidation in samples prepared at lower temperatures. The strong decrease of the residual resistivity with increasing $T_{s}$ agrees with these

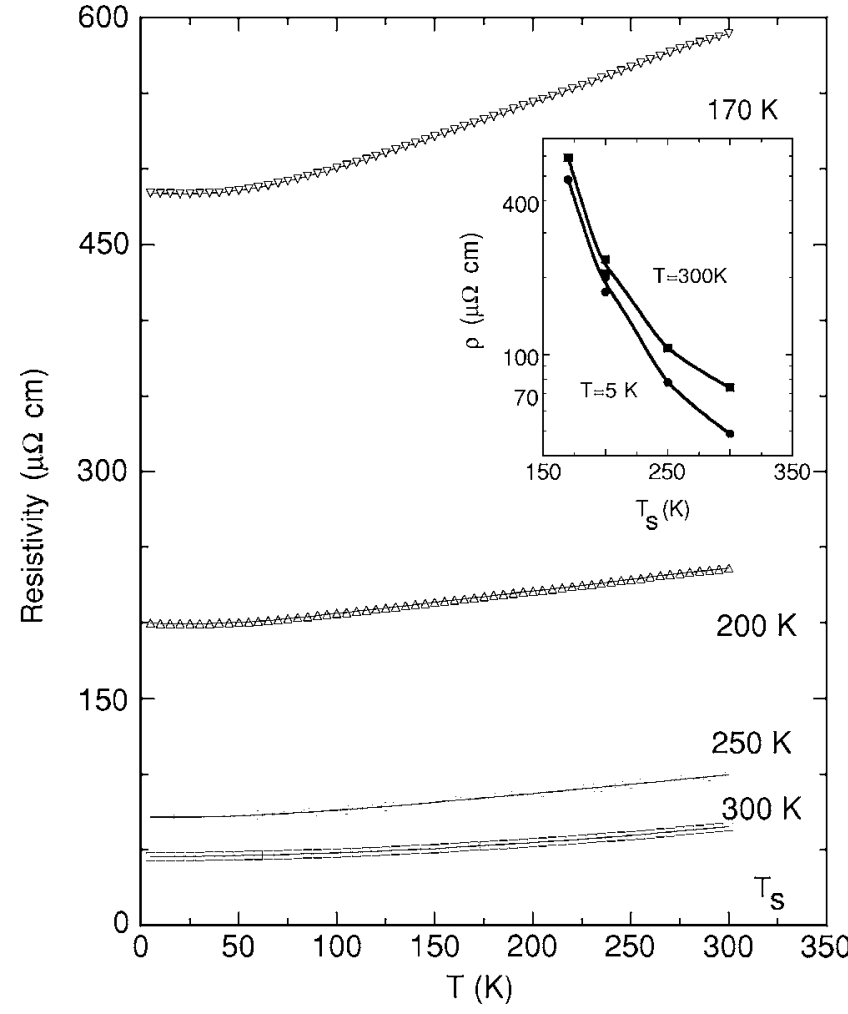

FIG. 1. Resistivity data points versus temperature for the oxidized $\mathrm{Fe}$ films grown at the substrate temperature in the range $170 \mathrm{~K} \leqslant T_{s} \leqslant 300 \mathrm{~K}$. The inset shows the resistivity at 5 and $300 \mathrm{~K}$ as a function of $T_{s}$. The solid lines are guides to the eye.

studies. Smaller grain size favors more effective oxidation and, consequently, more resistive behavior ensues. Table I displays, among other values, the room-temperature resistivities for our four films and those for Fe epitaxial film and for bulk iron. The effect of the granularity and of the oxidation in the films can be clearly appreciated.

The transverse magnetoresistance (MR) and the Hall resistivity are plotted in Figs. 2 and 3, respectively, as a function of the magnetic field. At $T=5 \mathrm{~K}$, both of these quantities follow the magnetization behavior in films shown in Fig. 4. The resistivity first decreases with increasing field and, at fields larger than approximately $2 \mathrm{~T}$, it saturates. Magnetoresistance values, which we find for films, are less than $1 \%$. A similar behavior is observed in the $\rho_{H}(H)$ plot in Fig. 3. The saturation field, estimated from MR and AHE measurements, becomes smaller in films prepared at lower temperatures; $H_{\text {sat }}$ varies from $\approx 2 \mathrm{~T}$ for $T_{s}=300 \mathrm{~K}$ to $\approx 1.2 \mathrm{~T}$ for $T_{s}=170 \mathrm{~K}$. Features, characteristic of AMR, are observed in the transverse MR at fields lower than $1 \mathrm{~T}$. In addition, the MR curves seem to be slightly asymmetric. We ascribe these to a slight deviation of the magnetic field from the normal to the plane of film.

Although $\rho_{H}(H)$ varies as $M(H)$ does in the oxidized Fe films we have studied, its amplitude varies with $T$ and $T_{s}$ in a completely different way. The saturation magnetization $M_{\text {sat }}$ for samples grown at the same substrate temperature $T_{s}$ is higher at $5 \mathrm{~K}$ than at room temperatures. In addition, $M_{\text {sat }}$ increases as $T_{s}$ increases. It is shown in the inset of Fig. 4. 
TABLE I. Electrical-transport and magnetic parameters obtained for oxidized iron films $(\mathrm{Fe} / \mathrm{FeO})$ used in this study. The literature values for epitaxial Fe films and bulk Fe are also given. $T_{s}$ is the deposition temperature, $\rho(300 \mathrm{~K})$ is the resistivity at $300 \mathrm{~K}, R R R$ stands for $\rho(300 \mathrm{~K}) / \rho(5 \mathrm{~K}), R_{o}$ and $R_{s}$ are the ordinary and anomalous Hall coefficients at room temperature, respectively, $\Delta \rho / \rho$ is the value of the longitudinal anisotropic magnetoresistance at $300 \mathrm{~K}, M_{s}$ is the saturation magnetization, and $H_{c}(300 \mathrm{~K})$ is the room-temperature in-plane coercive field.

\begin{tabular}{lcccccccc}
\hline \hline & $\begin{array}{c}T_{s} \\
\mathrm{~K}\end{array}$ & $\begin{array}{c}\rho(300 \mathrm{~K}) \\
\mu \Omega \mathrm{cm}\end{array}$ & $R R R$ & $\begin{array}{c}R_{o}(300 \mathrm{~K}) \\
10^{-10} \mathrm{~m}^{3} / \mathrm{C}\end{array}$ & $\begin{array}{c}R_{s}(300 \mathrm{~K}) \\
10^{-10} \mathrm{~m}^{3} / \mathrm{C}\end{array}$ & $\begin{array}{c}\Delta \rho / \rho \\
\%\end{array}$ & $\begin{array}{c}M_{s} \\
\mathrm{emu} / \mathrm{cm}^{3}\end{array}$ & $\begin{array}{c}H_{c}(300 \mathrm{~K}) \\
\mathrm{Oe}\end{array}$ \\
\hline $\mathrm{Fe} / \mathrm{FeO}^{\mathrm{a}}$ & 170 & 586 & 1.23 & & 690 & 0.10 & 725 & 73 \\
$\mathrm{Fe} / \mathrm{FeO}^{\mathrm{a}}$ & 200 & 235 & 1.20 & & 202 & 0.15 & 840 & 143 \\
$\mathrm{Fe} / \mathrm{FeO}^{\mathrm{a}}$ & 250 & 98 & 1.36 & & 35.6 & 0.20 & 940 & 118 \\
$\mathrm{Fe} / \mathrm{FeO}^{\mathrm{a}}$ & 300 & 64 & 1.52 & 1.9 & 20.8 & 0.20 & 1120 & 126 \\
$\mathrm{Fe}$ epitaxial & & 10 & 8.7 & 0.5 & 5.9 & 0.10 & 1700 & $\simeq 50$ \\
$\mathrm{Fe}$ bulk & & 9.7 & $\geqslant 90$ & 0.248 & 6.33 & 0.2 & 1700 & $\simeq 10$ \\
\hline \hline
\end{tabular}

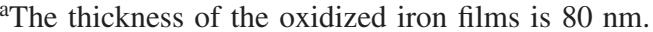

${ }^{\mathrm{b}}$ The parameters for the Fe epitaxial film (of $\approx 80 \mathrm{~nm}$ ) are from Refs. 11 and 12 .

${ }^{\mathrm{c}}$ The parameters for the bulk Fe are from Ref. 13.

On the other hand, Fig. 3 shows that the values of $\rho_{H}$ at $H>H_{\text {sat }}$ decreases as $T$ decreases or $T_{s}$ increases. Such behavior is brougth about by the resistivity variations: (i) $\rho$ is larger for films grown at lower temperatures and (ii) for films grown at the same temperature, $\rho$ increases with increasing $T$. In Fig. 5 we plot the anomalous Hall coefficient $R_{s}=\rho_{H} / 4 \pi M$ versus total resistivity at $T=5$ and $300 \mathrm{~K}$. We find that $R_{s}$ is roughly proportional to the square of the total resistivity, as predicted by the side-jump scattering model. ${ }^{6}$ This is reasonable, since the residual resistivity is high in our samples. A similar variation of AHE has been found before in epitaxial Fe films. ${ }^{11}$ The length of the side-jump amounts to approximately $2 \times 10^{-10} \mathrm{~cm}$ in our

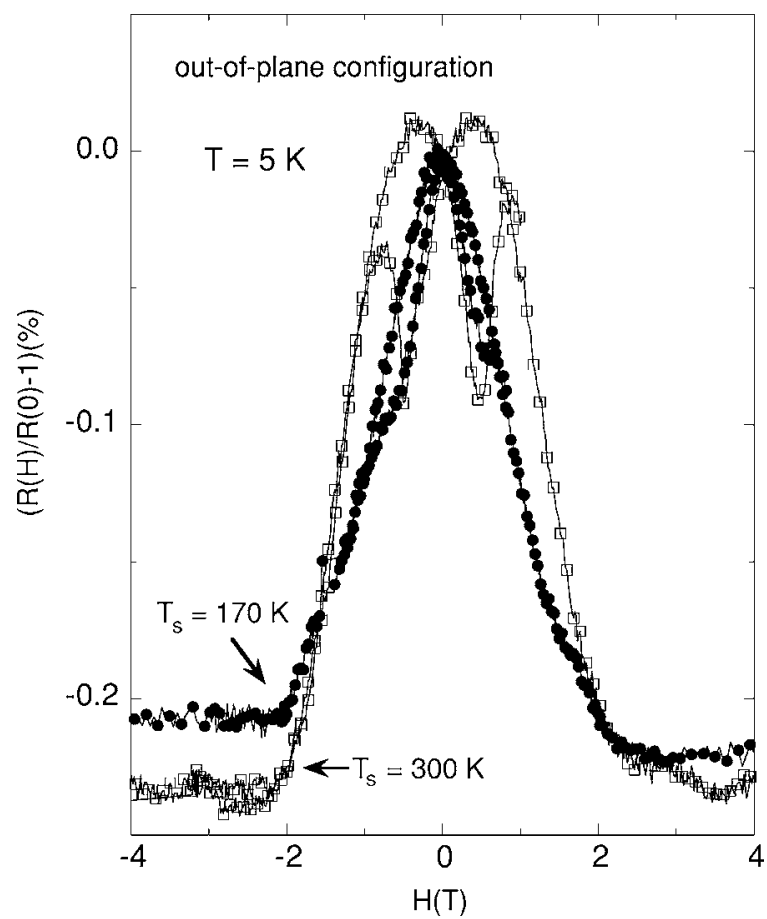

FIG. 2. Magnetoresistance of two films $\left(T_{s}=170\right.$ and $\left.300 \mathrm{~K}\right)$ as a function of the applied magnetic field at $T=5 \mathrm{~K}$. films, which is one order of magnitude smaller than the one-band estimate in ferromagnetic materials. ${ }^{6}$ A more realistic band structure model is supposed to bring experiment and theory in agreement. ${ }^{6}$ The high-field slope of the Hall resistivity gives the ordinary Hall coefficient. We find $R_{o} \approx-1.9 \times 10^{-10} \mathrm{~m}^{3} \mathrm{C}^{-1}$ in the films we have studied, which is very close to values previously found in Fe films. ${ }^{11}$

Interestingly, neither the transverse magnetoresistance nor the Hall resistivity show any common features with the hysteresis loops observed in the out-of-plane magnetization. We would expect some perpendicular anisotropy in our films

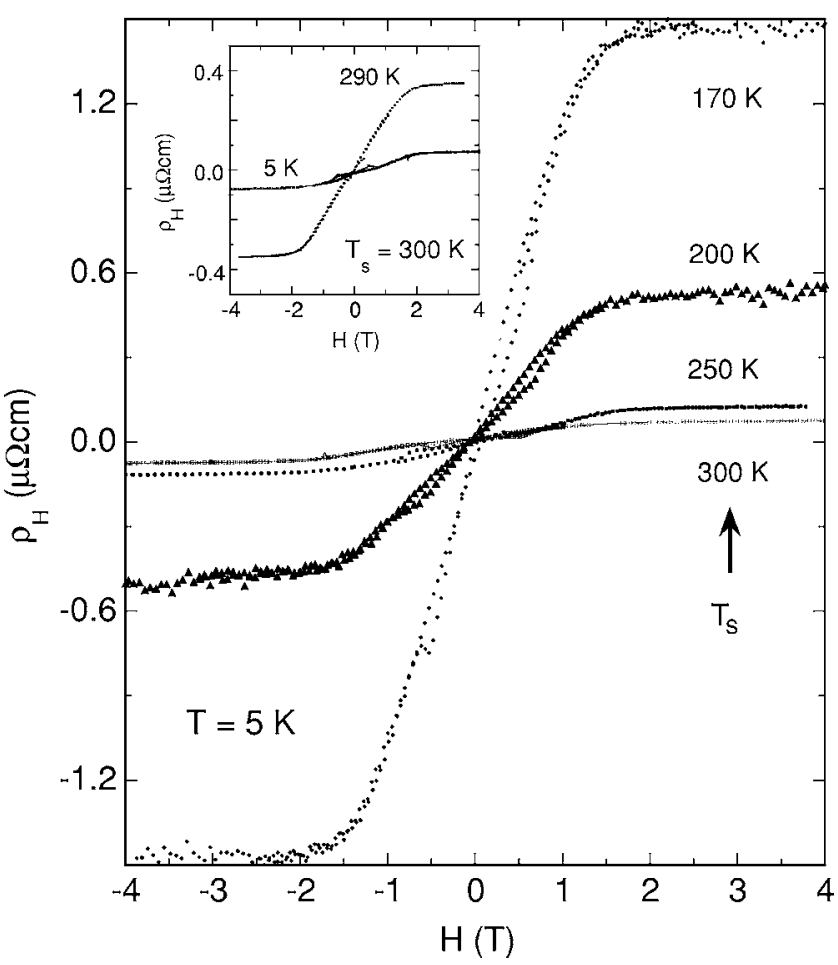

FIG. 3. Hall resistivity of the Fe films, prepared at various $T_{s}$, as a function of the applied magnetic field at $T=5 \mathrm{~K}$. The inset shows the Hall resistivity for the $T_{s}=300 \mathrm{~K}$ film at $T=5$ and $290 \mathrm{~K}$. 


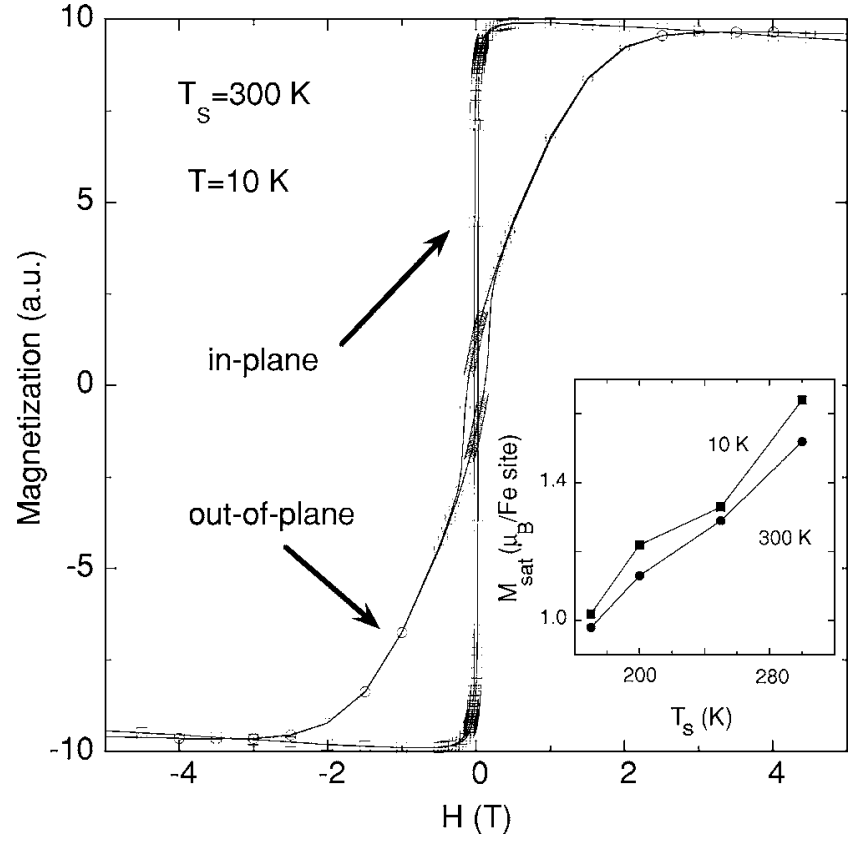

FIG. 4. Out-of-plane and in-plane magnetization loops at $T=10 \mathrm{~K}$ for the $T_{S}=300 \mathrm{~K} \mathrm{film}$. The inset exhibits the saturation magnetization as a function of $T_{s}$ for $T=10$ and $300 \mathrm{~K}$.

since the magnetostatic interactions favor out-of-plane moments for columnar growth. However, as discussed below, we find only a strong in-plane anisotropy which we attribute to the surface spins of nanocrystalline grains. As their magnetic moments tend to be normal to the surface, they confine the total magnetization to the film plane. An extrinsic mag-

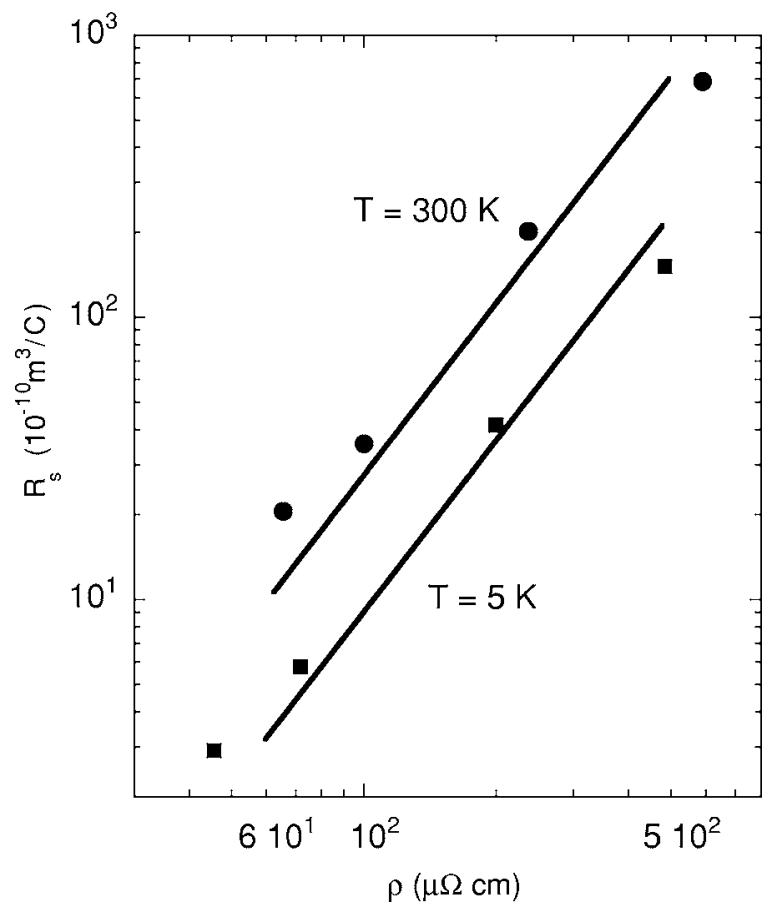

FIG. 5. Anomalous Hall coefficient as a function of the longitudinal resistivity for the films at $T=5$ and $300 \mathrm{~K}$. The solid lines show a $\rho^{2}$ dependence.

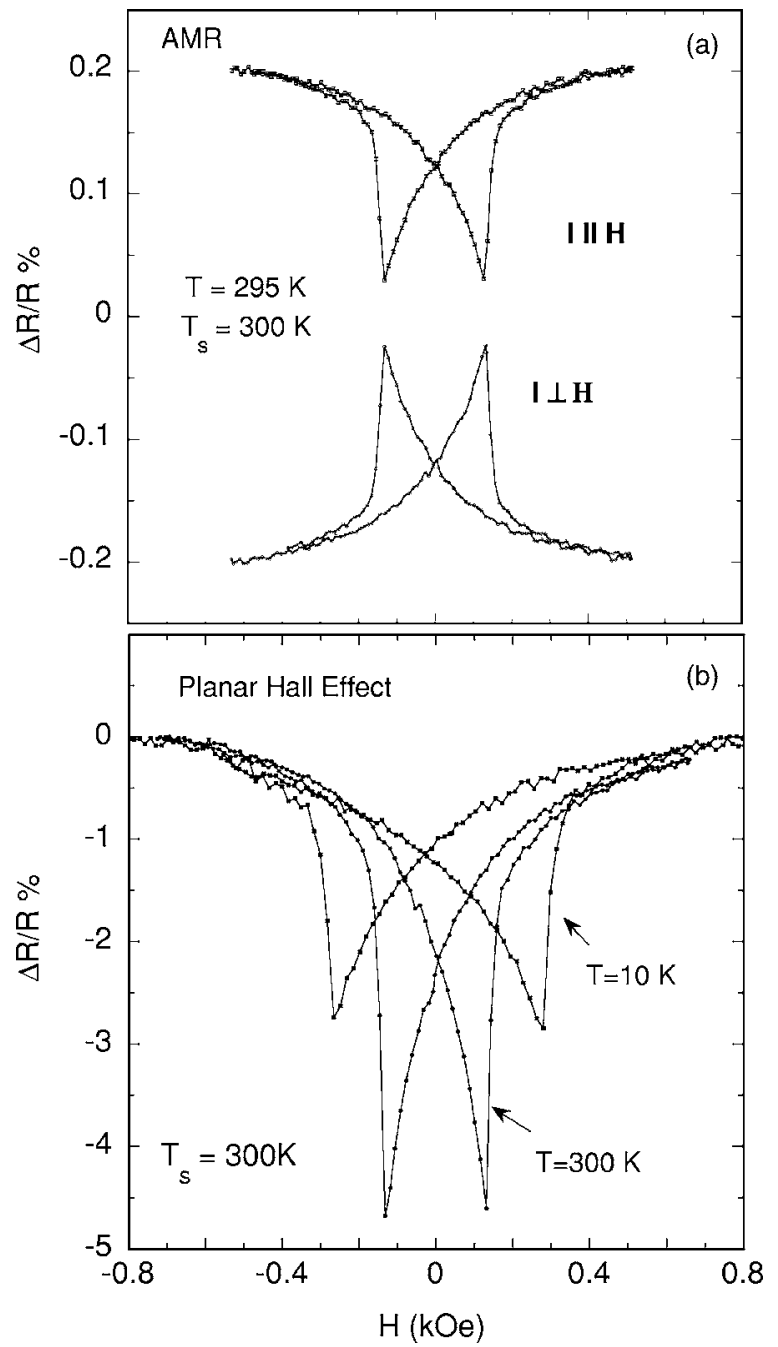

FIG. 6. (a) Magnetoresistance measurements in the out-of-plane and in-plane configurations for the $T_{s}=300 \mathrm{~K}$ film. (b) Planar Hall effect for the same film at $T=10$ and $300 \mathrm{~K}$. The peaks correspond to the coercive field. The solid lines are to the eye.

netic property such as the coercivity depends drastically on morphology and texture of the film, ${ }^{14}$ to which transport properties are less sensitive. Therefore, it may happen that mostly reversible transverse magnetoresistance and Hall effect are observed while the magnetization behavior shows irreversible behavior.

\section{B. Anisotropic magnetoresistance and magnetization dynamics}

Now we turn to the AMR measurements and magnetization dynamics. How the magnetoresistance, for the in-plane longitudinal and transversal configurations, varies with magnetic field is shown in Fig. 6(a). The AMR value is $\sim 0.2 \%$ at $295 \mathrm{~K}$, and it does not vary significantly with temperature. In the diagonal in-plane configuration (current at $\sim 45^{\circ}$ to the magnetic field), MR variations are much larger, of the order of a several percent, as shown in Fig. 6(b). We find MR values higher than $20 \%$ for some films in this configuration, without taking special care for contact placing. Peaks in MR appear when the distribution of magnetic domains shows 


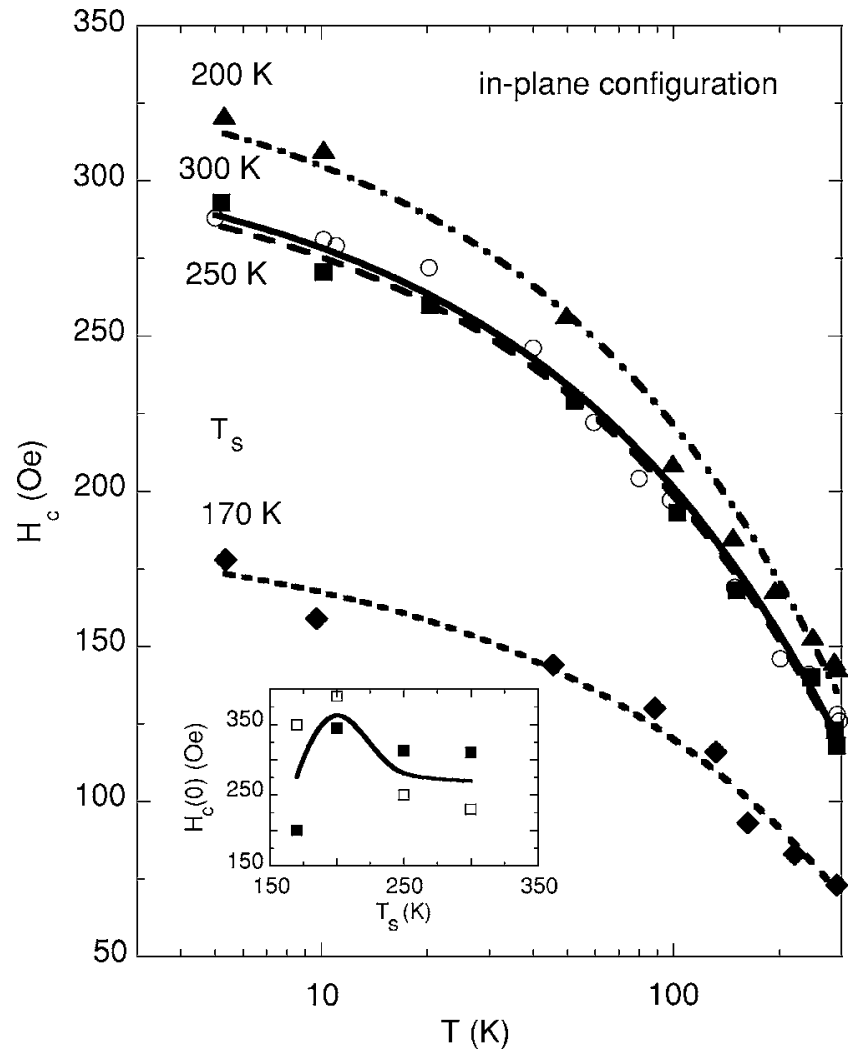

FIG. 7. Temperature variation of the switching field in the oxidized Fe films for the in-plane configuration. The solid and dashed lines are fits to experimental points. The inset show $H_{c}(0)$, obtained from fits, as a function of $T_{s}$ (closed points) and $H_{c}(10 \mathrm{~K})$, obtained from magnetization measurements, for samples from the same batch (open points).

maximum disorder. This occurs approximately at the coercive field. We plot the values of $H_{c}$, obtained from diagonal AMR measurements, versus temperature in Fig. 7. The switching field increases with decreasing temperature as expected for a thermally activated process. All experimental data points can be fitted with the expression $H_{c}(T)$ $=H_{c}(0)\left[1-\left(T / T_{0}\right)^{1 / 2}\right]$, where $H_{c}(0)$ and $T_{0}$ are fitting parameters. This relation is often used to describe coherent magnetization reversal in a system of uniaxial noninteracting particles with the anisotropy axes aligned parallel to the applied magnetic field. ${ }^{15,16}$ The solid and dashed lines in Fig. 7 are for the temperature variations of $H_{c}$ we have calculated using the above relation. The agreement with the experimental data points is quite good. The parameter $T_{0}$ is almost independent of $T_{s}$ and is approximately $770 \mathrm{~K}$.

In thermally activated magnetization reversal an energy barrier $\Delta E \propto k_{B} T_{0}$, where $k_{B}$ is the Boltzmann's constant, is overcome. ${ }^{17}$ Several factors, such as magnetocrystalline and magnetostatic anisotropies or interparticle interactions, contribute to $\Delta E$. As pointed out above, the surface anisotropy of crystalline grains is important in the system we have studied. Although a cylindrically symmetric anisotropy would average to zero in the film plane, ${ }^{18}$ surface inhomogeneities give rise to an energy barrier. ${ }^{19}$ Anisotropy of magnetic ions in sites of low symmetry is rather large. ${ }^{20}$ Values between 1 and $4 k_{B}$ /cation are obtained for the surface anisotropy of $\mathrm{Fe}$ ions from EPR measurements. ${ }^{18}$ They are much larger than the bulk Fe anisotropy $\left(0.05 k_{B} /\right.$ cation $)$. To estimate the surface energy barrier, we note that the total number of magnetic atoms in one columnar grain is about $5 \times 10^{3}$ for the sizes we have. A large fraction of these atoms is located on the surface of the grain. For random spin orientations, $E_{s} \approx K_{s} \sqrt{N_{S}}$. Here, $E_{s}, K_{s}$, and $N_{s}$ are the surface barrier energy, the surface anisotropy constant, and the number of surface spins, respectively. Since $N_{S}$ is of the order of $1500, E_{s} \approx 160 \mathrm{~K}$. The actual surface of crystalline grains can, however, be quite irregular and can therefore lodge many more ions leading to higher values of the surface energy barrier. The value of $E_{s}$ is much larger than bulk crystalline anisotropy energy of single domain grains. In addition, dipolar and exchange interactions contribute to the total anisotropy energy. We estimate the dipole-dipole interactions to be of the order of $10^{2} \mathrm{~K}$ for nearest-neighbor grains. ${ }^{21}$ Intergrain exchange interactions may not be very important as there are oxide layers between grains. We find from our fit that $T_{0}$ does not vary with the growth temperature $T_{s}$. Since the size of crystalline grains decreases with decreasing $T_{s}$, a total surface anisotropy becomes larger. However, dipole-dipole interactions are weaker for smaller grains. ${ }^{14}$ Therefore, the total anisotropy $\left(\propto k_{B} T_{0}\right)$ may remain constant.

The variation of the zero-temperature coercive field $H_{c}(0)$ with $T_{s}$ is shown in the inset of Fig. 7. $H_{c}(0)$ is largest for $T_{s}=200 \mathrm{~K}$, and decreases slightly as $T_{s}$ increases. Since $H_{c}$ is of the order of $K_{\text {eff }} / M_{s}$, such behavior agrees with the above conclusions. The value of $H_{c}(0)$ for the film prepared at $T_{s}=170 \mathrm{~K}$ is abnormally low, which may be attributed to its partially amorphous nature. The behavior found for $H_{c}(0)$ agrees qualitatively with the variation of $H_{c}\left(T_{s}\right)$, which was obtained from measurements of in-plane magnetization at $T=10 \mathrm{~K}$, for the films from the same batch (shown in the inset of Fig. 7). In order to get a feeling for the variation of the coercive field with $T_{s}$, we recall that the coercivity of a single-domain particulate material decreases when particle size becomes smaller than some value. ${ }^{22}$ In $\mathrm{Ag}$ coated $\mathrm{Fe}$ particles this occurs at approximately $170 \AA .{ }^{1}$ Crystalline grains in our films are smaller than this but they are slightly oxidized. Magnetic interactions between the metallic core and the metal-oxide shell, which vary strongly with temperature, often lead to large values of the coercivity. We expect a competition between these two effects in our system. However, at present it is difficult to quantify either of these effects. Further research is needed for this purpose.

\section{CONCLUDING REMARKS}

Summarizing, we find that the electrical transport properties of naturally oxidized Fe thin films depend strongly on their growth temperature $T_{s}$. The resistivity increases by more than one order of magnitude as $T_{s}$ decreases from 300 to $170 \mathrm{~K}$. We attribute this variation to a more effective oxidation of crystalline Fe grains in films prepared at lower substrate temperatures. The rise of the anomalous Hall resistivity in films grown at lower $T_{s}$ comes mainly from variation of the resistivity with $T_{s}$. Side-jump scattering seems to 
be responsible for such behavior. We find very large magnitudes of the anomalous Hall coefficient in granular samples studied. This may be interesting for the design of novel fieldsensing devices. The coercive fields, obtained from the anisotropic magnetoresistance measurements, follow a squareroot temperature dependence. Such a variation follows from the Stoner-Wohlfarth model for a system of weakly interacting single-domain particles that relax through the Neel mechanism. A large surface uniaxial anisotropy of crystalline grains would lead to a similar behavior. However, dipolar interactions may well be important in our system.

\section{ACKNOWLEDGMENTS}

We acknowledge support from Ministerio de Ciencia y Tecnología of Spain through Grants No. MAT02/166 and MAT2003/01880. We thank Professor Juan Bartolomé for stressing the role dipolar interactions play here.
*Email address: jolanta@unizar.es

${ }^{1}$ Nanomaterials: Synthesis, Properties and Applications, edited by A. S. Edelstein and R. C. Cammarata (Institute of Physics, Bristol, 1996).

${ }^{2}$ J. Smit, Physica (Amsterdam) 16, 612 (1951).

${ }^{3}$ T. R. McGuire and R. I. Potter, IEEE Trans. Magn. 11, 1018 (1975).

${ }^{4}$ R. I. Potter, Phys. Rev. B 10, 4626 (1974).

${ }^{5}$ J. Smit, Physica (Amsterdam) 21, 877 (1955); 24, 39 (1958).

${ }^{6}$ L. Berger, Phys. Rev. B 2, 4559 (1970).

${ }^{7}$ R. Karplus and J. M. Luttinger, Phys. Rev. 95, 1154 (1954).

${ }^{8}$ F. Jiménez-Villacorta, A. Muñoz-Martín, and C. Prieto, J. Appl. Phys. 96, 6224 (2004).

${ }^{9}$ A. Gerber, A. Milner, L. Goldshmit, M. Karpovski, B. Lemke, H.-U. Habermeier, and A. Sulpice, Phys. Rev. B 65, 054426 (2002).

${ }^{10}$ F. Jiménez-Villacorta, A. Muñoz-Martín, and C. Prieto, Nucl. Instrum. Methods Phys. Res. B 238, 340 (2005).

${ }^{11}$ R. Schad, P. Beliën, G. Verbanck, V. V. Moshchalkov, and Y. Bruynseraede, J. Phys.: Condens. Matter 10, 6643 (1998).
${ }^{12}$ J. Yu, U. Rüdiger, A. D. Kent, L. Thomas, and S. S. P. Parkin, Phys. Rev. B 60, 7352 (1999).

${ }^{13}$ M. Rubinstein, F. J. Rachford, W. W. Fuller, and G. A. Prinz, Phys. Rev. B 37, 8689 (1988).

${ }^{14}$ R. Skomsky and J. M. Coey, in Permanent Magnetism (Institute of Physics, Bristol, 1999).

${ }^{15}$ C. P. Bean and J. D. Livingston, J. Appl. Phys. 40-S, 1205 (1959).

${ }^{16}$ J. García-Otero, A. J. García-Bastida, and J. Rivas, J. Magn. Magn. Mater. 189, 377 (1998).

${ }^{17}$ M. P. Sharrock, IEEE Trans. Magn. Mag-20, 754 (1984).

${ }^{18}$ R. H. Kodama and A. E. Berkowitz, Phys. Rev. B 59, 6321 (1999).

${ }^{19}$ L. Néel, J. Phys. Radium 15, 225 (1954).

${ }^{20}$ W. Low, Paramagnetic Resonance in Solids (Academic, New York, 1960), p. 33.

${ }^{21}$ G. Brown, M. A. Novotny, and Per Arne Rikvold, Phys. Rev. B 64, 134422 (2001).

${ }^{22}$ C. Chen, O. Kitakami, and Y. Shimada, J. Appl. Phys. 84, 2184 (1998). 